\title{
Impacto de las estrategias de aprendizaje en educación a distancia sobre el desempeño estudiantil en un curso introductorio de la ciencia del suelo
}

\author{
Impact of Learning Strategies in Distance Education on Student Performance in an \\ Introductory Course of Soil Science
}

Impacto das estratégias de ensino à distância no desempenho de estudantes de um curso introdutório de Ciências do Solo

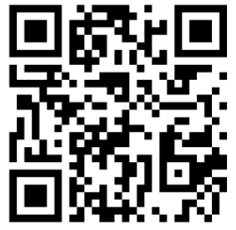

Leticia Jiménez-Álvarez

Universidad Técnica Particular de Loja

Departamento de Ciencias Biológicas

Loja, Ecuador

Isjimenez@utpl.edu.ec

(D) https://orcid.org/0000-0002-7933-1368

Natacha Fierro-Jaramillo

Universidad Técnica Particular de Loja

Departamento de Ciencias Biológicas

Loja, Ecuador

ndfierro@utpl.edu.ec

(D) http://orcid.org/0000-0001-6309-4276

Pablo Quichimbo-Miguitama

Universidad de Cuenca

Facultad de Ciencias Agropecuarias

Cuenca, Ecuador

pablo.quichimbo@ucuenca.edu.ec

iD https://orcid.org/0000-0002-6108-9091

Daniel Capa-Mora

Universidad Técnica Particular de Loja

Departamento de Ciencias Biológicas

Loja, Ecuador

edcapa@utpl.edu.ec

https://orcid.org/0000-0002-9843-0388

Recibido • Received • Recebido: 07 / 06 / 2019

Corregido • Revised • Revisado: 15 / 01 / 2021

Aceptado • Accepted • Aprovado: 24 / 02 / 2021 
Resumen: El propósito de la investigación es determinar, si las estrategias de aprendizaje influyeron en la participación y rendimiento del estudiantado de una asignatura de la ciencia del suelo para la modalidad a distancia. Se utilizó la participación y calificaciones de 783 e studiantes de una asignatura de gestión ambiental de una Universidad en el sur del Ecuador. Se organizaron los datos en cuatro periodos comprendidos entre el 2012 y 2018 y se analizaron con estadística descriptiva (medias y frecuencias), tomando en cuenta las estrategias aplicadas en cada periodo, como foros, chats, cuestionarios, trabajos y evaluaciones. Las estrategias sincrónicas y asincrónicas utilizadas influyen en la participación del estudiantado, pero no siempre en su rendimiento; además, el mayor número de actividades enviadas no siempre resulta provechoso; por ello, es clave priorizar y clarificar las actividades que se envíen al estudiantado, con una adecuada planificación y explicación de ellas. Las características únicas de la ciencia del suelo, sumadas al desafío de la enseñanza en modalidad a distancia, constituyen un verdadero reto por lo que continuar investigando los aspectos de la educación de esta ciencia, contribuirá a mejorar el rendimiento académico del estudiantado.

Palabras claves: Estrategia pedagógica; enseñanza online, suelo, motivación.

Abstract: This research aims to determine if learning strategies influenced the participation and performance of students from a Soil Science course in distance modality. The participation and qualifications of 783 students from an Environmental Management course of a university in southern Ecuador were used. The data were organized in four periods between 2012 and 2018 and analyzed with descriptive statistics (means and frequencies), considering the strategies implemented in each period, such as forums, chats, questionnaires, works, and evaluations. The implemented synchronous and asynchronous strategies influence the students' participation but not always their performance. Furthermore, the greater number of activities sent is not always beneficial. For this reason, it is essential to prioritize and clarify the activities sent to students with adequate planning and explanation of them. The unique characteristics of Soil Science, added to the challenge of distance learning, constitute a real challenge, so continuing to do research on education aspects of this science will contribute to improving the students' academic performance.

Keywords: Pedagogical strategy; online teaching; soil; motivation.

Resumo: O objetivo da pesquisa é verificar se as estratégias de aprendizagem influenciaram a participação e o desempenho de estudantes de uma disciplina de Ciências do Solo para a modalidade a distância. Utilizou-se a participação e qualificação de 783 estudantes de uma disciplina de Gestão Ambiental de uma Universidade do sul do Equador. Os dados foram organizados em quatro períodos entre 2012 e 2018 e analisados com estatística descritiva (médias e frequências), levando em consideração as estratégias aplicadas em cada período, como fóruns, chats, questionários, trabalhos e avaliações. As estratégias síncronas e assíncronas utilizadas influenciam na participação dos estudantes, mas nem sempre em seu desempenho, além disso, a maioria das atividades enviadas nem sempre foram aproveitadas; por esse motivo, é fundamental priorizar e esclarecer as atividades que são enviadas aos estudantes, com planejamento e explicação adequada das mesmas. As características singulares da Ciência do Solo, somadas ao modo do ensino a distância, constituem um verdadeiro desafio. Portanto, continuar investigando os aspectos educacionais dessa ciência contribuirá para melhorar o desempenho acadêmico dos estudantes.

Palavras-chave: Estratégia pedagógica; ensino on-line, solo, motivação. 


\section{Introducción}

A nivel de educación superior (universitaria) y dentro del contexto ecuatoriano, existen tres modalidades, la educación presencial, semipresencial y la educación a distancia. Cuando nos referimos a modalidad presencial, generalmente, en el proceso de enseñanza, se incluye una parte práctica en campo y laboratorio en escenarios reales (Hughes y Overton, 2009); sin embargo, es un desafío incluir las mismas estrategias con estudiantes de modalidad a distancia. Esta suele ser criticada por no involucrar directamente a sus estudiantes en el ámbito práctico, por lo tanto, esas estrategias deben ser compensadas con otras actividades, que hagan vínculos entre la teoría y la práctica, y contribuyan a favorecer una formación integral del estudiantado del plan de estudios, por lo que incluye el desarrollo de TIC (UNESCO, 2002). Cabe indicar que esta modalidad de estudios permite la preparación de miles de estudiantes que, debido a sus obligaciones de trabajo, familiares o de residencia, no pueden asistir a la universidad de manera presencial.

Por otra parte, el personal docente no es nativo digital sino inmigrante digital, lo que se convierte en una limitante al momento de plantear nuevas estrategias pedagógicas a través del entorno virtual de aprendizaje (Albertini González, 2017; Amador y Mederer, 2013); además, constituye un desafío llegar al estudiantado, debido a que son población nativa digital, la cual se desmotiva con las conferencias extensas, con la instrucción lenta y muy seria, y que sigan un orden determinado; en cambio, prefieren los gráficos a los textos, descargar música, vídeos, chatear, trabajan siempre en red (Prensky, 2001).

A nivel global se ha visto una tendencia creciente al uso de nuevas metodologías (Shirani Bidabadi et al., 2016), las que contienen una combinación de componentes interactivos y no interactivos. El utilizar estrategias como los libros electrónicos, mensajes de texto, simulaciones, podcasting, wikis, blogs, animaciones, vídeos interactivos (Kim y Bonk, 2006; Lockwood y Daniel, 1997; Mamo et al., 2004) son algunas de las opciones que plantean algunos estudios; lo más importante es que las estrategias promuevan la interacción, retroalimentación relevante y el aprendizaje que debe ayudar a que el estudiantado construya su propio aprendizaje (Gill y Rengel, 2013).

La educación a distancia está ganando espacio en la educación universitaria (Driscoll et al., 2012), en donde el factor económico, las competencias pedagógicas, técnicas del personal instructor, tecnologías online, idiosincrasia del profesorado y estudiantes son algunos de los factores que afectan a este tipo de educación (García Aretio, 1999; Kim y Bonk, 2006). Esta modalidad de educación tiene un crecimiento relativamente reciente, por lo que aún se debe investigar sobre las estrategias para el aprendizaje del estudiantado (Driscoll et al., 2012), considerando que cada curso es diferente y único en el aprendizaje a distancia (Song et al., 2004); más aún en el caso de la ciencia del suelo. 
http://doi.org/10.15359/ree.25-2.6

http://www.una.ac.cr/educare

educare@una.ac.cr

No se puede enseñar eficazmente siguiendo principios de enseñanza genéricos, porque la naturaleza única del suelo requiere enfoques especiales y diferentes maneras de pensar (Field et al., 2011), lo que motiva a seguir investigando.

Ante lo expuesto, el propósito de esta investigación es determinar, si las estrategias de aprendizaje influyeron en la participación del estudiantado y en su rendimiento académico dentro de la asignatura Manejo Sustentable del Suelo, en un contexto de educación a distancia, presentado como un estudio de caso, en una universidad ecuatoriana.

\section{Metodología}

Como parte del pensum de la Carrera de Gestión Ambiental de la Universidad Técnica Particular de Loja -UTPL- (Sede central localizada en la ciudad de Loja al sur del Ecuador), se ofertó a nivel de pregrado la asignatura troncal de carrera de la Ciencia del Suelo para la modalidad a distancia, en donde participaron estudiantes de diversas localidades rurales y urbanas de todo el Ecuador, pertenecientes a diferente edad, género, estado civil, cultural, social y económica. Para esta investigación se incluyeron varios ciclos académicos (cada ciclo con una duración de 5 meses aproximadamente), distribuidos en cuatro periodos académicos de acuerdo con las estrategias desarrolladas.

- Periodo uno: comprendió tres ciclos académicos, desde octubre 2012 hasta febrero 2014, con un total de 147 estudiantes, este periodo incluye como estrategias o actividades académicas a desarrollar por el estudiantado, únicamente el trabajo a distancia y la evaluación presencial (ver detalle Figura 1).

- Periodo dos: incluyó desde abril 2014 hasta febrero 2016, con 439 estudiantes en total, aquí se incluyeron, adicionales al trabajo a distancia y a la evaluación presencial, otras actividades opcionales como el foro, el chat y la videocolaboración, cada una de ellas con una valoración de un punto.

- El periodo tres: comprendió el ciclo académico abril-agosto 2016, con 147 estudiantes, aquí se incluyen las actividades del periodo dos, se elimina la videocolaboración para ser reemplazada por la aplicación de cuestionarios.

- Periodo cuatro: abarcó el ciclo octubre 2017-febrero 2018, con 50 estudiantes, en donde se utilizan las actividades del periodo tres, pero todas de forma obligatoria.

Se analizaron estos cuatro periodos debido a la diversidad de estrategias utilizadas para la enseñanza; ya que, antes del 2012, la asignatura tenía otro nombre y algunos contenidos académicos se modificaron y, después del 2019 se rediseñó la Carrera, por ello, no se consideraron 
http://doi.org/10.15359/ree.25-2.6

http://www.una.ac.cr/educare educare@una.ac.cr

otros periodos académicos. Durante el periodo de investigación, el mismo docente impartió la asignatura y preparó el material de clase. En este trabajo no se presenta la participación del estudiantado en tutorías, debido a que la participación era muy baja en todos los periodos y era difícil el registro a través de la plataforma y por vía telefónica.

Figura 1: Estrategias utilizadas en los cuatro periodos de un curso de las Ciencias del Suelo en modalidad abierta y a distancia en una Universidad del Sur de Ecuador, en resaltado se encuentran las actividades obligatorias

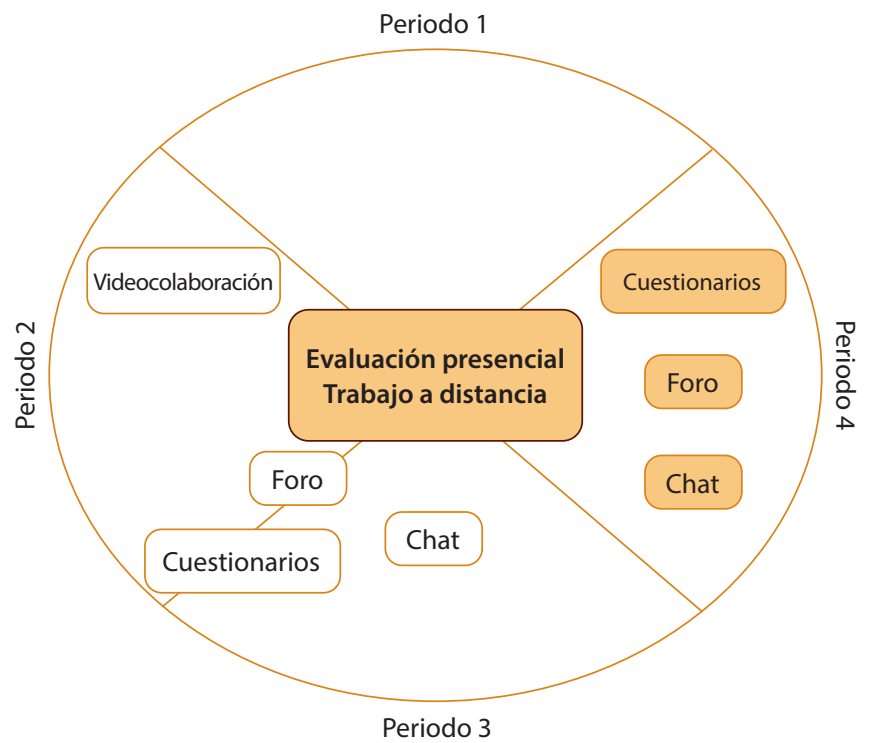

Nota: Elaboración propia.

Las estrategias presentadas se basaron en los lineamientos de modalidad abierta y a distancia de la UTPL, tanto las obligatorias como opcionales. Todas estas estrategias se realizan a través de la plataforma del entorno virtual de aprendizaje (EVA), donde es imprescindible que el estudiantado ingrese a la plataforma virtual, y cargue sus tareas en la fecha prevista de acuerdo con los calendarios establecidos por la UTPL. En el caso de la evaluación final que demanda presencialidad, el estudiantado tuvo que acudir a los centros UTPL a nivel nacional en la fecha establecida por la Universidad (Tabla 1). 
http://doi.org/10.15359/ree.25-2.6

http://www.una.ac.cr/educare

educare@una.ac.cr

Tabla 1: Estrategias planteadas en el componente de las Ciencia del Suelo

\begin{tabular}{|c|c|}
\hline Estrategia & Característica \\
\hline Foro & $\begin{array}{l}\text { Se plantea un tema referente al plan de estudios y el alumnado dispone aproximadamente } \\
\text { de una semana para responder. Se programan dos foros por ciclo académico distribuidos } \\
\text { para cada bimestre de estudio. }\end{array}$ \\
\hline Chat & $\begin{array}{l}\text { Se plantea un tema de los estudiados y el alumnado debe participar en la fecha planteada, } \\
\text { el chat se desarrolla durante } 1 \text { hora por cada bimestre. Son dos chats por ciclo académico. }\end{array}$ \\
\hline Videocolaboración & $\begin{array}{l}\text { Se discute sobre un tema de los estudiados, el alumnado participa contestando a las } \\
\text { preguntas que plantea el personal docente, esta actividad dura alrededor de una hora. Son } \\
\text { dos videocolaboraciones por ciclo académico. }\end{array}$ \\
\hline Cuestionarios & $\begin{array}{l}\text { Son } 4 \text { cuestionarios por bimestre, en cada uno de ellos se plantea entre } 5 \text { a } 10 \text { preguntas y } \\
\text { tienen alrededor de } 15 \text { minutos para contestarlos. }\end{array}$ \\
\hline Trabajo a distancia & $\begin{array}{l}\text { Consistió en alrededor } 40 \text { preguntas objetivas (dicotómicas o de opción múltiple) con } \\
\text { una valoración de } 2 \text { puntos. También incluyó una parte de ensayo, en la que se plantearon } \\
\text { preguntas de análisis, en lo posible que se complemente con parte de campo. Por periodo } \\
\text { académico son dos trabajos a distancia (bimestre } 1 \text { y } 2 \text { ). }\end{array}$ \\
\hline $\begin{array}{l}\text { Evaluación } \\
\text { presencial }\end{array}$ & $\begin{array}{l}\text { Dos evaluaciones presenciales, cada una de ellas con } 40 \text { preguntas, si el estudiantado no } \\
\text { se presenta a rendirla, debe dar evaluación de recuperación. Son dos evaluaciones por } \\
\text { periodo académico. }\end{array}$ \\
\hline Tutorías & $\begin{array}{l}\text { Las tutorías se las imparte por dos horas a la semana y son opcionales para el alumnado, } \\
\text { aquí pueden realizar preguntas, sugerencias o discusión sobre los temas de la asignatura, } \\
\text { se imparten a través de la plataforma o por vía telefónica. }\end{array}$ \\
\hline Otras estrategias & Vídeos cortos, presentaciones en PowerPoint, artículos, información, frases motivacionales. \\
\hline
\end{tabular}

Nota: Elaboración propia.

El rendimiento académico fue evaluado usando una escala ordinal con cinco categorías: excelente, corresponde en la escala cuantitativa de 39-40 puntos; muy bueno de 35-38; bueno de 31-34; regular de 28-30 y deficiente menor a 28 puntos, se aprueba con al menos 28 puntos (Reglamento interno del sistema de evaluación estudiantil UTPL, 2017).

\section{Análisis de datos}

Para determinar si las estrategias de aprendizaje influyeron en la participación de las actividades planteadas y en el rendimiento del estudiantado en los cuatro periodos académicos, se utilizaron análisis estadísticos descriptivos (medias y frecuencias). Para determinar la participación del género se promedió a estudiantes hombres y mujeres que se habían inscrito y se expresó en porcentaje. Para evaluar la participación del estudiantado en las actividades síncronas como el chat, videocolaboración y la evaluación presencial; y en actividades asíncronas 
http://doi.org/10.15359/ree.25-2.6

como los cuestionarios, el foro y el trabajo a distancia, se presentaron los datos también en porcentaje. En el caso del rendimiento académico se promedió cada periodo (4 periodos) y se comparó entre sí, debido a que en cada periodo se utilizaron diferentes estrategias de aprendizaje, los datos se expresan en porcentaje para un mejor análisis comparativo por periodo académico y calificación cualitativa obtenida.

\section{Resultados y discusión}

En los resultados se puede observar que hubo un mayor porcentaje de inscripción en el curso de las Ciencias del Suelo por parte del género femenino (Figura 2), a excepción de los otros periodos, lo que indica que las mujeres podrían estar viendo el estudio en modalidad a distancia como una clara opción de superación personal y profesional; esto, posiblemente, por las múltiples actividades que deben desarrollar, sumadas a las de ser estudiante. Según manifiesta la UNESCO (2002), en programas a distancia, las mujeres suelen alcanzar un porcentaje significativo sobre todo cuando no existen otras formas de desarrollo convencional para la preparación en una carrera profesional.

Figura 2: Porcentaje de inscripción por género en la asignatura Manejo Sustentable del Suelo en Modalidad Abierta y a Distancia en una Universidad del Sur de Ecuador

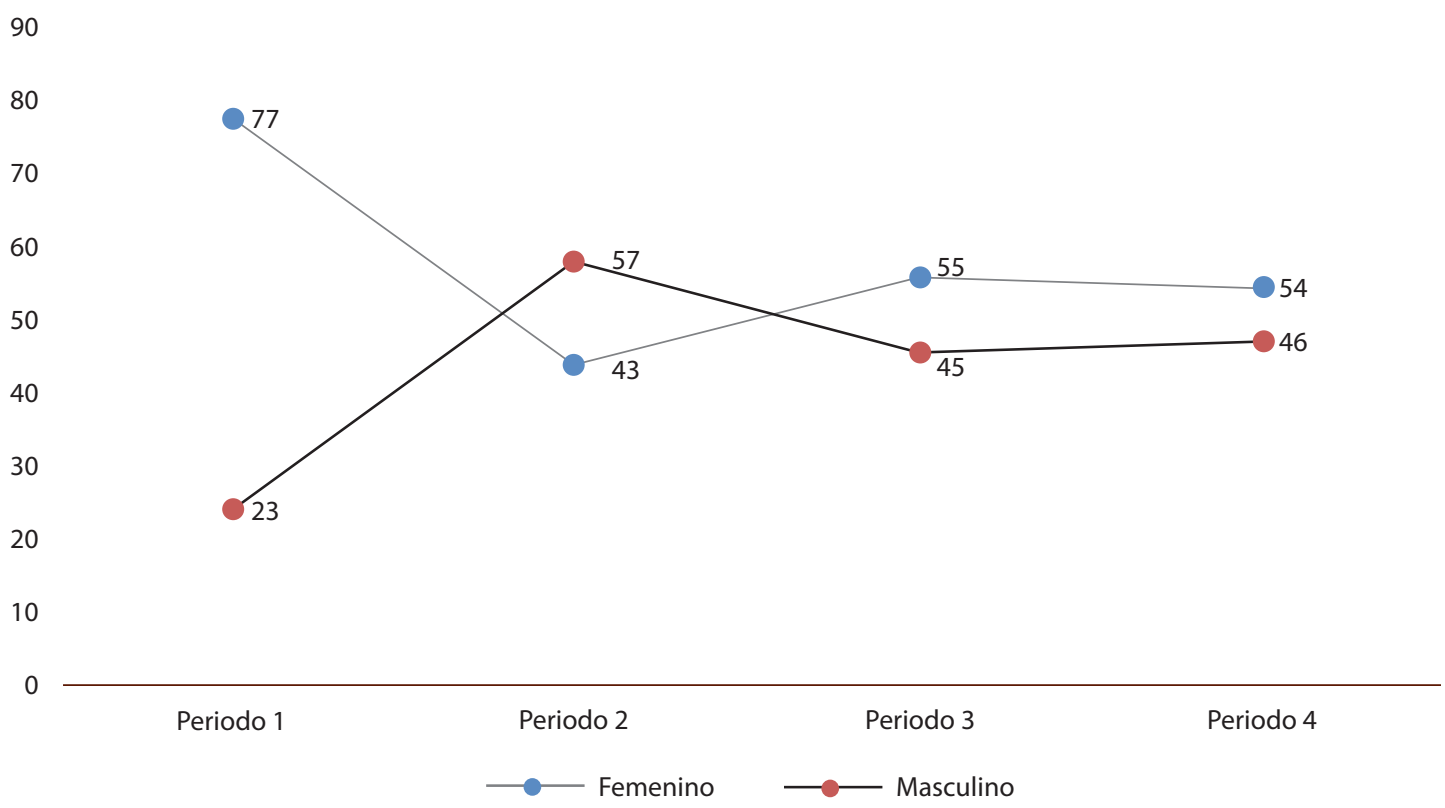

Nota: Elaboración propia. 
http://doi.org/10.15359/ree.25-2.6

http://www.una.ac.cr/educare

educare@una.ac.cr

Sin embargo, si consideramos esta información por ubicación geográfica, en el mismo estudiantado, observamos que la tendencia de estudio es similar entre los dos géneros (Jiménez Álvarez et al., 2018), lo que permite intuir que a medida que pasa el tiempo, tanto hombres como mujeres ven esta opción de estudio por igual.

En cuanto a las estrategias propuestas, los resultados de esta investigación indican que la participación mantiene la tendencia a través del tiempo (cuatro periodos), el trabajo a distancia y la evaluación fueron las que tuvieron mayor participación frente a las otras actividades, debido a que si no lo desarrollaban o no se presentaban reprobarían el curso, a pesar de haber cumplido con el trabajo a distancia y las actividades opcionales; el hecho de que el trabajo y la evaluación sean obligatorias y sean las de mayor puntaje, constituye una forma de motivación para el estudiantado, por tanto, este factor define la permanencia del estudiantado en el sistema educativo en modalidad a distancia (Moncada Mora, 2014).

Las estrategias como el foro, chat, cuestionarios y videocolaboración, en ese orden, son las que tuvieron menor participación. En el periodo uno no existieron alternativas adicionales al trabajo y a la evaluación presencial. En el periodo dos y tres, a pesar de ser actividades opcionales en las que podrían ganar puntaje, tuvieron una participación menor al 70\%; el foro fue la actividad preferida (Figura 3), seguida del chat y, finalmente, de la videocolaboración; al ser estos dos últimos recursos síncronos, dan menor flexibilidad al estudiantado para su participación, característica clave en este tipo de educación (Pérez Martínez y Tlaczani Conde, 2012).

Esto podría ser debido a que fue la primera vez que se instauró este tipo de prácticas, y al ser opcionales, el alumnado prefiere no realizarlas, ya sea por cuestiones de tiempo, de trabajo o simplemente por no estar familiarizado con estas actividades. Los nuevos cambios implementados pueden generar incertidumbre y rechazo al inicio, por lo que es necesario, primero, familiarizar al estudiantado con las reformas que se vayan a implementar.

En el periodo cuatro en el cual se presentaron actividades síncronas y asíncronas obligatorias, los cuestionarios (actividad 1 y 2), la participación del estudiantado fue muy alta, a diferencia de los chats en donde la participación del estudiantado fue la más baja. Curry et al. (2007) mencionan que las tareas y las sesiones de chat son una de las mejores ventanas para que quien instruye conozca la comprensión de los materiales de la asignatura de sus estudiantes; sin embargo, en este caso se aprecia que la participación en el chat fue menor al $60 \%$, esto podría ser porque un gran número de las personas que estudian a distancia trabajan y conectarse virtualmente con sus docentes en el horario establecido dificulta su participación (Jarret et al., 2011), debido a que los horarios de los chats son establecidos previamente en el horario de la tutoría, factor limitante para la participación de este estudiantado. 
http://doi.org/10.15359/ree.25-2.6

Figura 3: Porcentaje de participación del estudiantado inscrito en cada periodo, en las actividades utilizadas en la asignatura Manejo Sustentable del Suelo en modalidad abierta y a distancia en una Universidad del Sur de Ecuador

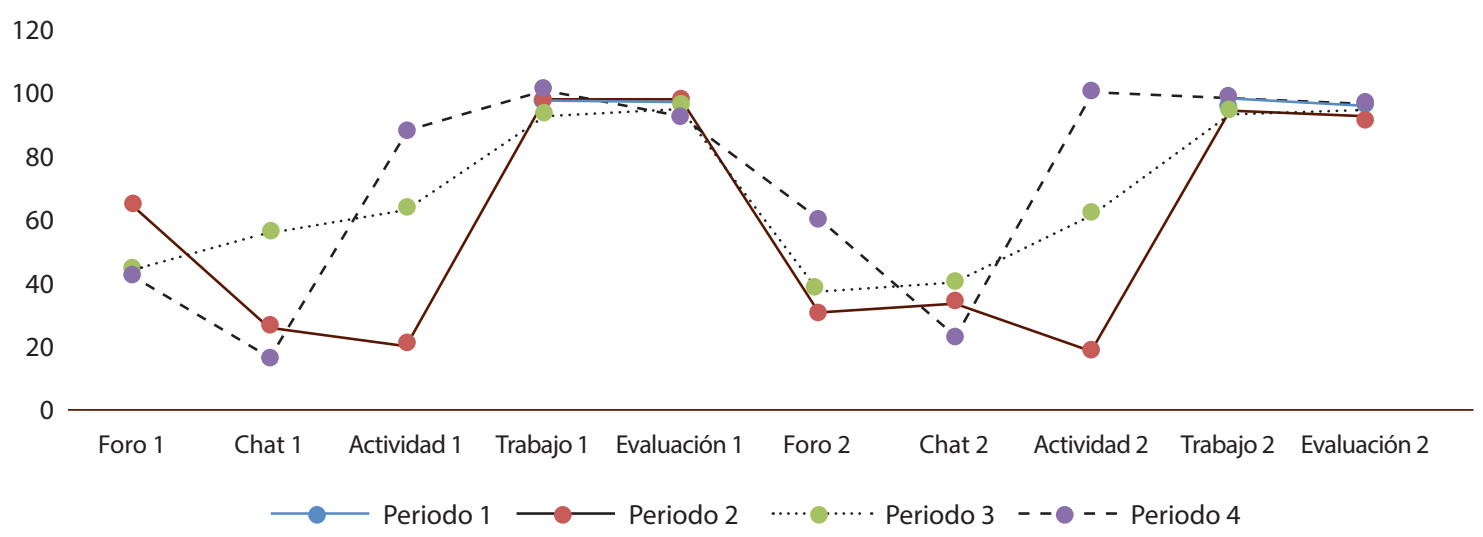

Nota: Elaboración propia.

Aún menor fue la participación en las videocolaboraciones, actividad que se realizó únicamente en el periodo dos (actividad 1), con apenas el 20\%. Seguramente el estudiantado se siente intimidado por la persona docente, ya que la comunicación, además de ser síncrona, era visual y en tiempo real, y el estudiantado no está acostumbrado a la interacción diaria que se tiene en la educación presencial. Algunos de los alumnos y las alumnas comentaron que no tuvieron buena conexión a internet, lo que no les permitió participar.

Opciones para incrementar la participación en actividades síncronas como el chat o la videocolaboración podrían ser realizar esta actividad en horarios más accesibles para el alumnado, por ejemplo, en horas de la noche o los fines de semana. Otra alternativa es realizar al menos dos chats académicos o dos videocolaboraciones por cada bimestre, para duplicar las posibilidades de participación. Una tercera opción sería que el estudiantado participe en actividades síncronas o asíncronas con temáticas similares y con calificación en una de ellas, para que pueda elegir en cuál de las dos actividades participar.

Existen experiencias en educación a distancia relacionadas con el tema. Así tenemos que, en la Universidad La Florida, se ofertó el curso: Introducción a la Ciencia del Suelo a través de videoconferencias y se incluyeron también una parte de laboratorio (Curry et al., 2007). Otra experiencia es la de Murphy (2000) en un curso general de suelo, en donde el estudiantado a distancia interactuó con el personal instructor por videoconferencia durante una hora cada semana $y$, a pesar de ser menos estudiantes que en otras modalidades y la oportunidad de mayor interacción, la experiencia no fue del todo positiva. Lozano et al. (2015) sugieren incluir más actividades sincrónicas, sin embargo, nuestros resultados no apoyan esto para la modalidad a distancia. De acuerdo con las características de la asignatura y con los estilos de aprendizaje 
http://doi.org/10.15359/ree.25-2.6

http://www.una.ac.cr/educare

educare@una.ac.cr

del estudiantado (Jiménez Álvarez et al. 2019), también se podrían considerar laboratorios virtuales y herramientas para grabar, editar, compartir audios, imágenes o vídeos, infografías, muros virtuales, presentaciones animadas, cuentos virtuales, revistas digitales.

De las actividades planteadas inicialmente como opcionales (periodo dos y tres) y luego como obligatorias (periodo cuatro), las que mayor éxito mostraron fueron los cuestionarios, probablemente porque se plantearon cuatro por bimestre y por ser una actividad que permite al estudiantado desarrollarla de acuerdo con sus condiciones, además de tener un mayor tiempo para realizarlos (aproximadamente una semana), esta mayor participación podría asociarse a la flexibilidad, característica clave del estudiantado de modalidad a distancia, para acceder al curso de acuerdo con el tiempo disponible y al lugar en donde está (Mamo et al., 2011).

Según Song et al. (2004), la comunicación asíncrona permite escribir más cuidadosamente sobre sus ideas y es muy importante la flexibilidad, lo que nos indica que, es posible que el tiempo fuera insuficiente, debido a que en los dos periodos tuvieron una participación entre el 30 y $65 \%$, ya que gran número del estudiantado no revisa su entorno virtual de aprendizaje semanalmente, incluso existen casos de estudiantes que trabajan en campamentos sin acceso a internet, y están ausentes dos o tres semanas. Este es un punto importante que se debe mejorar para los futuros cursos.

Otra actividad asíncrona mencionada por Egbert (2000) es el estudio de casos reales, considerados como una transición entre la teoría y la práctica, en donde existe un problema basado en el aprendizaje, cuyo objetivo del trabajo a distancia consiste en que el estudiantado realice una parte como trabajo de campo del lugar donde reside y con base en ello efectuar una discusión. Según Field et al. (2017) y Hartemink (2015), para que se incremente el conocimiento de la ciencia del suelo, el aprendizaje debe tener énfasis en aplicaciones prácticas y tener lugar en su contexto físico y conceptual, cuanto más cerca esté el estudiantado de su realidad, mejor estará equipado para enfrentar cada vez más desafíos globales.

También Ramasundaram et al. (2005) dicen que los ejercicios de laboratorio y la observación de campo es clave para los cursos de ciencias de la tierra y ciencias ambientales, pero es muy complicado para cada asignatura incorporar las prácticas necesarias, las cuales son limitadas por el tiempo, el dinero y la distancia. Lo importante es compensar, de cierta manera, la ausencia de salidas de campo y prácticas de laboratorio, importantes para este componente y carrera (Curry et al., 2007). Por ejemplo, Motavalli et al. (2003) sugieren escribir un contenido ambiental relacionado con el tema edáfico, que puede ser considerado un método de aprendizaje activo como herramienta eficaz para que el estudiantado aprenda. De igual manera, debemos buscar en lo posible que los trabajos asignados se conviertan en una interesante opción para que el estudiantado a distancia revise el material (Curry et al., 2007), lo que ayudará a que incremente su conocimiento en la materia.

Algunos estudios sugieren que se realicen trabajos grupales y que sean seleccionados al azar, que incluyan tareas que no pueden ser realizadas de manera individual (Jarret et al., 2011; 
http://doi.org/10.15359/ree.25-2.6

Kim y Bonk, 2006); sin embargo, para nuestra realidad y para las condiciones individuales que presenta el estudiantado, por ser de sitios muy diversos de todo el país, ahora mismo será difícil aplicar esta sugerencia.

Los estudios mencionados anteriormente y los nuestros muestran que no existe una estrategia única para aplicarla al estudiantado, estas dependerán de las características de la materia, titulación, condiciones del medio, de conectividad, etc.

La educación a distancia requiere mayor reflexión que la educación tradicional (Song et al., 2004). Es importante que tanto el personal docente como el alumnado estén motivados y abiertos a nuevas estrategias de enseñanza y aprendizaje (Jarret et al., 2011), porque el proceso de la educación es dinámico. Un método de enseñanza adecuado ayuda al estudiantado y lo motiva a aprender (Shirani Bidabadi et al., 2016), eso dependerá de las condiciones grupales e individuales de sus aprendices.

Si el personal docente que enseña la Ciencia del Suelo es entusiasta y se divierte enseñando, eso se transmitirá a sus estudiantes y probablemente despertará fascinación por el recurso suelo (Hartemink et al., 2014).

El estudiantado se enfocó, principalmente, en las actividades de mayor peso en el puntaje, las cuales corresponden al trabajo a distancia y a la evaluación presencial, lo que aseguró que el mayor porcentaje de estudiantes apruebe la materia.

Figura 4: Porcentaje de participación del estudiantado según su rendimiento en modalidad abierta y a distancia en una Universidad del Sur de Ecuador

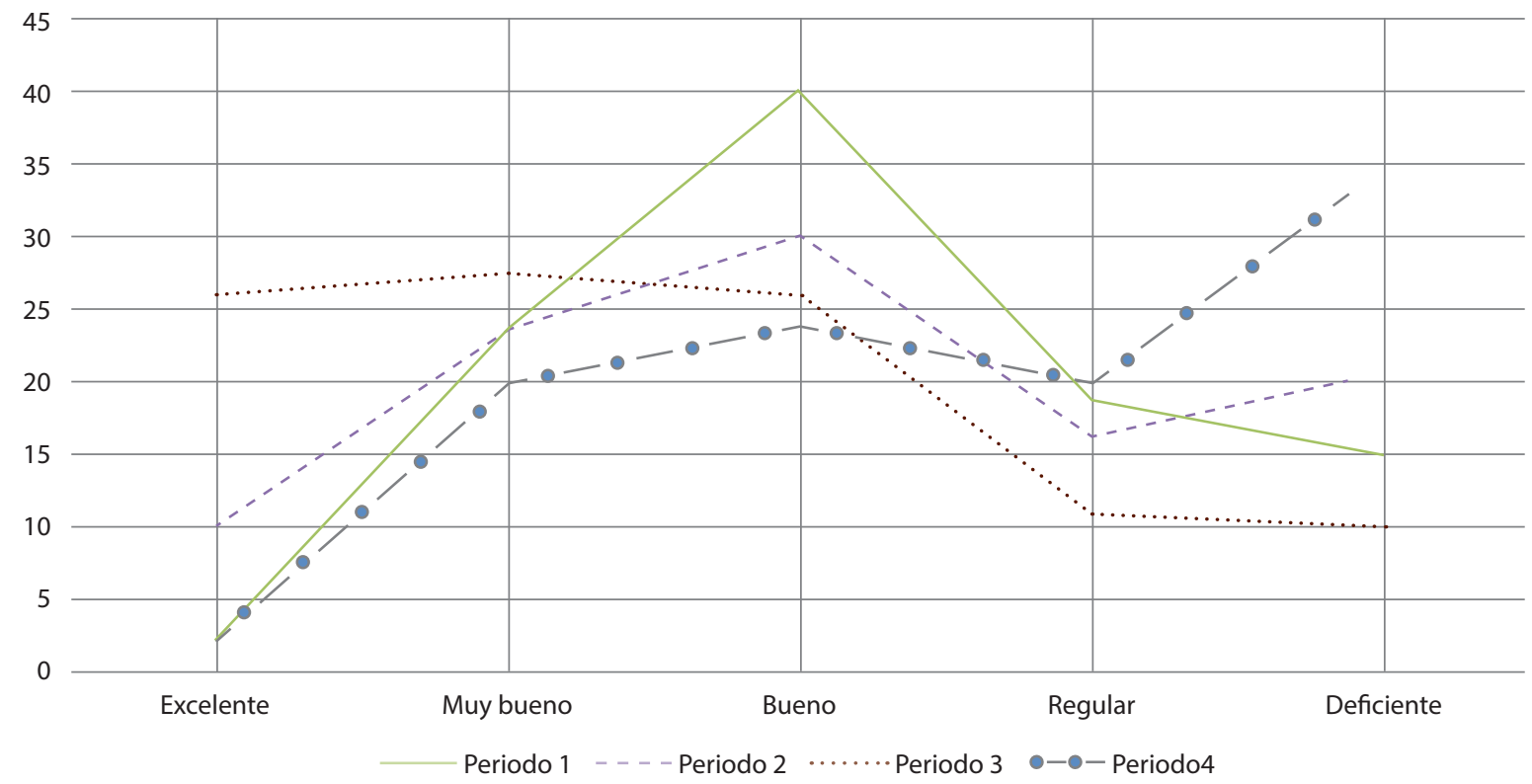

Nota: Elaboración propia. 
http://doi.org/10.15359/ree.25-2.6

http://www.una.ac.cr/educare

educare@una.ac.cr

Las estrategias utilizadas influyeron, en algunos casos, en el rendimiento académico. Así se observa en la Figura 4, en el periodo tres, se utilizaron foros, chats y cuestionarios y en este se logró el porcentaje más alto con notas excelentes, a diferencia del periodo 1, en donde no se utilizó ninguna de esas estrategias y se obtuvo el porcentaje más bajo. En el caso del periodo cuatro, en donde las actividades fueron obligatorias, se obtuvo menor porcentaje de estudiantes con notas de excelente y el mayor porcentaje con nota deficiente (menor a 28 puntos). Hubiéramos esperado que el periodo uno presente el promedio más bajo; sin embargo, mostró un promedio de 31,45 puntos a pesar de utilizar solo dos recursos, además del uso menor de la tecnología y problemas de conectividad que, probablemente, eran mayores al periodo cuatro, debido a que la tecnología ha jugado y continúa jugando un papel en el desarrollo y expansión de la educación en línea (Kim y Bonk, 2006).

En cambio, en el periodo cuatro se asumía que se obtendría el promedio más alto, y se logró un promedio de 26,74 puntos que resultó ser el promedio más bajo, a pesar de utilizar varios recursos de manera obligatoria y una evaluación continua. No obstante, el periodo tres presentó el promedio más alto con 33,62 puntos, lo que nos muestra que no siempre el mayor número de estrategias utilizadas se refleja en un mayor promedio del estudiantado, pues factores como el grado de dificultad de las tareas, de los foros, chats, etc. podrían incrementar la comprensión del estudiantado, lo que se vería expresado en el rendimiento final. Datos diferentes obtuvieron Moreno et al. (2017), quienes mencionan que la evaluación continua apoya al estudiantado a superar, de manera positiva, una asignatura y mejora los conocimientos de la materia.

Se evidencia que el rendimiento académico de las personas participantes de cursos de las ciencias del suelo de modalidad a distancia está asociado a diferentes factores, entre ellos, las estrategias utilizadas. Por lo tanto, es imprescindible la labor docente y pedagógica para propiciar escenarios y estrategias de enseñanza y aprendizaje que promuevan el interés, la motivación y el empoderamiento del estudiantado, sin necesidad de apuntar a que sean selectivos únicamente a las actividades con mayor peso o que son calificadas, sino que, más bien, se integren y participen de todas las actividades sincrónicas y asincrónicas que contribuyen a una preparación consecutiva hasta llegar a su evaluación presencial.

\section{Conclusiones}

Con base en los resultados del presente trabajo, a manera de conclusión, se evidencia que, para la asignatura de Manejo Sustentable del Suelo durante los cuatro periodos, no existe una relación directa entre el género del estudiantado con la participación en el curso. En relación con el rendimiento académico, las diferentes estrategias y actividades e información multimodal, algunas de carácter síncrono y asíncrono, influyeron en el rendimiento académico del estudiantado. La mayor diversidad de herramientas y estrategias, en algunos casos, 
afecta positivamente el aprendizaje reflejado en un incremento del rendimiento académico del estudiantado; sin embargo, un número exagerado de herramientas para estudiantes de modalidad a distancia puede desanimar y ofuscar al alumnado por el limitado tiempo que dispone. Por ello, es importante priorizar y clarificar las actividades que se envíen a estudiantes de esta modalidad, con una adecuada planificación y explicación y se deben tomar en cuenta las particularidades propias que caracterizan esta población estudiantil.

Este trabajo se desarrolló como un estudio de caso que contribuiría al conocimiento de los aspectos de la educación de la ciencia del suelo, un tema que ha sido alentado a nivel mundial por la importancia que representa el manejo sostenible del recurso suelo.

\section{Declaración de Material complementario}

Este artículo tiene disponible, como material complementario:

-La versión preprint del artículo en https://doi.org/10.5281/zenodo.3661311

\section{Referencias}

Albertini González, F. A. (2017). Avances, desafíos e impacto de la educación superior virtual en Paraguay 2015-2017. Revista ScientiAmericana, 4(1), 1-23. https://www.uamericana.edu. py/revistacientifica/index.php/scientiamericana/article/view/237/231

Amador, J. A. y Mededer, H. (2013). Migrating successful student engagement strategies online: Opportunities and challenges using Jigsaw groups and problem-based learning. Journal of Online Learning and Teaching, 9(1), 89-105. https://digitalcommons.uri.edu/nrs facpubs/42/

Curry, S., Grunwald, S. y Vimala, N. (Eds). (2007). Distance education. Myakka, 7(3), 1-8. https:// soils.ifas.ufl.edu/media/soilsifasufledu/sws-main-site/pdf/newsletters/fall-2007.pdf

Driscoll, A., Jicha, K., Hunt, A. N., Tichavsky, L. y Thompson, G. (2012). Can online courses deliver in-class results? A comparison of student performance and satisfaction in an online versus a face-to-face introductory sociology course. Teaching Sociology, 40(4), 312-331. https:// doi.org/10.1177/0092055X12446624

Egbert, J. (2000). [Reseña del libro Teaching and learning a distance: Foundations of distance education, por M. Simonson, S. Smaldino, M. Albright y S. Zvacek]. Language Learning \& Technology, 4(1), 16-19. https://www.Iltjournal.org/item/2311 
http://doi.org/10.15359/ree.25-2.6

http://www.una.ac.cr/educare

educare@una.ac.cr

Field, D. J., Koppi, A. J., Jarrett, L. E., Abbott, L. K., Cattle, S. R., Grant, C. D., McBratney, A. B., Menzies, N. W. y Weatherley, A. J. (2011). Soil science teaching principles. Geoderma, 167-168, 9-14. https://doi.org/10.1016/j.geoderma.2011.09.017

Field, D. J., Yates, D., Koppi, A. J., McBratney, A. y Jarret, J. (2017). Framing a modern context of soil science learning and teaching. Geoderma, 289, 117-123. https://doi.org/10.1016/j. geoderma.2016.11.034

García Aretio, L. (1999). Historia de la educación a distancia. Revista lberoamericana de Educación a Distancia, 2(1), 11-40. https://doi.org/10.5944/ried.2.1.2084

Gill, J. K. y Rengel, Z. (2013). Designing an online lecture in the discipline of soil science. Teaching and Learning Forum, 1-9. https://clt.curtin.edu.au/events/conferences/tlf/tlf2013/refereed/ gill.pdf

Hartemink, A. E. (2015). On global soil science and regional solutions. Geoderma Regional 5, 1-3. https://doi.org/10.1016/j.geodrs.2015.02.001

Hartemink, A. E., Balks, M. R., Chen, Z.-S.., Drohan, P., Field, D. J., Krasilnikov, P., Lowe, D. J., Rabenhorst, M., van Ress, K., Schad, P., Schipper, L. A., Sonneveld, M. y Walter, C. (2014). The joy of teaching soil science. Geoderma, 217-218, 1-9. https://doi.org/10.1016/j. geoderma.2013.10.016

Hughes, I. y Overton, T. (2009). Key aspects of learning and teaching in experimental sciences. En H. Fry., S. Ketteride. y S. Marshall (Eds.), A Handbook for teaching and learning in higher education. Enhancing academic practice (pp. 226-245). Routledge. http://biblioteca.ucv. $\mathrm{cl} /$ site/colecciones/manuales u/A\%20Handbook\%20for\%20Teaching\%20and\%20 Learning\%20in\%20Higher\%20Education\%20Enhancing\%20academic\%20and\%20 Practice.pdf

Jarret, L. E., Field, D. J., Koppi, T., Koppittke, P. M., Abbott, L., Grant, C. D., McBratney, A. B., Menzies, N. W. y Weatherley, T. F. K. (2011). Guidelines for online learning in soil science: A synthesis of ideas from academics, students and employers. En Teaching for Diversity - Challenges and Strategies ACSME (pp. 175-180). University of Melbourne.

Jiménez Álvarez, L., Capa-Mora, E., Quichimbo Miguitama, P. y Fierro Jaramillo, N. (2018). Enseñanza de la introducción de la ciencia del suelo en modalidad a distancia en Ecuador. Revistas Ciencias Pedagógicas e Innovación, 6(1), 52-60. https://doi.org/10.26423/rcpi. v6i1.211

Jiménez Álvarez, L. S.,Vega, N.,Capa-Mora, E. D., Fierro Jaramillo, N. del C.y Quichimbo Miguitama, P. (2019). Estilos y estrategia de enseñanza-aprendizaje de estudiantes universitarios de la ciencia del suelo. Revistas Electrónica de Investigación Educativa, 21(e04), 1-10. https://doi. org/10.24320/redie.2019.21.e04.1935 
Kim, K.-J. y Bonk, C. J. (2006). The future of online teaching and learning in higher education. The survey says... Educause Quarterly, 4, 22-30. https://er.educause.edu/-/media/files/articledownloads/eqm0644.pdf

Lockwood, P. y Daniel, H. (1997). Computer-assisted teaching in soil science. Science of Soils, 2. http://hintze-online.com/sos/1997/Articles/Art5/art5.html

Lozano, Z., Ruiz, M. y Lozano, R. (2015). Experiencias en educación a distancia en ciencia del suelo. Información y documentación agrícola, 31, 67-72. https://www.researchgate.net/ publication/285152960 Experiencia en educacion a distancia en ciencia del suelo

Mamo, M., Ippolito, J. A., Kettler, T. A., Reuter, R. y McCallister, D., Morner, P., Husmann, D. y Blankenship, E. (2011). Learning gains and response to digital lessons on soil genesis and development. Journal of Geoscience Education, 59(4), 194-204. https://doi. org/10.5408/1.3651402

Mamo, M., Kettler, T., Husmann, D. y McCallister, D. (2004). Assessment of an on-line erosion lesson as a teaching tool in introductory soil science. NACTA Journal, 48(3), 47-52.

Moncada Mora, L. F. (2014). La integración académica de los estudiantes universitarios como factor determinante del abandono de corto plazo, un análisis en el sistema de educación superior a distancia del Ecuador. Red Iberoamericana de Estudios del Desarrollo, 17(2), 173196. https://doi.org/10.5944/ried.17.2.12683

Motavalli, P. P., Patton, M. D., Logan, R. A. y Frey, C. J. (2003). Promoting environmental writing in undergraduate soil science programs. Journal of Natural Resources and Life Sciences Education, 32(1), 93-99. https://doi.org/10.2134/jnrlse.2003.0093

Moreno, B., Ramos, C. y García, A. S. (2017). Efectos de las estrategias de enseñanza y aprendizaje universitarias en el rendimiento académico. Revista d'Innovació Docent Universitària, 9, 3953. https://revistes.ub.edu/index.php/RIDU/article/view/RIDU2017.9.4/20378

Murphy, T. H. (2000). An evaluation of a distance education course design for general soils. Journal of Agricultural Education, 41(3), 103-112. https://pdfs.semanticscholar.org/ce9f/1e a629aa6267afc5b1349bd337031e213794.pdf? ga=2.49161889.622051483.15953757751594467074.1583199411

Pérez Martínez, A. y Tlaczni Conde, E. (2012). Herramientas síncronas y asíncronas en educación a distancia. En Cuarto Congreso Virtual Iberoamericano de Calidad en Educación a Distancia (pp. 1-9). FLEAD; RIDEAD; REMAD; TUEDA, Asociación Venezolana de Educación a Distancia. http://eduqa2012.eduqa.net/eduqa2012/images/ponencias/eje4/4 3 PEREZ Alain TLACZANI_Esther_herramientas sincronas y asincronas en educacion a distancia.pdf 
http://doi.org/10.15359/ree.25-2.6

http://www.una.ac.cr/educare

educare@una.ac.cr

Prensky, M. (2001). Digital natives, digital inmigrants. On the Horizon, 9(5), 1-20. https:// www.marcprensky.com/writing/Prensky\%20-\%20Digital\%20Natives,\%20Digital\%20 Immigrants\%20-\%20Part1.pdf

Ramasundaram, V., Grunwald, S., Mangeot, A., Comerford, N. B.y Bliss, C. M. (2005), Development of an environmental virtual field laboratory. Computers \& Education, 45(1), 21-34. https:// doi.org/10.1016/j.compedu.2004.03.002

Shirani Bidabadi, N., Nasr Isfahani, A., Rouhollahi, A. y Khalili, R. (2016). Effective teaching methods in higher education: Requirements and barriers. Journal of Advances in Medical Education \& Professionalism, 4(4), 170-178.

Song, L., Singleton, E. S., Hill, J. R. y Koh, M. H. (2004). Improving online learning: Student perceptions of useful and challenging characteristics. The Internet and Higher Education, 7(1), 59-70. https://doi.org/10.1016/j.iheduc.2003.11.003

UNESCO. (2002). Aprendizaje abierto y a distancia. Consideraciones sobre tendencias, políticas y estrategias. Autor. http://unesdoc.unesco.org/images/0012/001284/128463s.pdf 